\title{
Spatio-temporal structure of the interaction between turbulence and flows at the L-H transition in a toroidal plasma
}

\author{
T. Estrada, ${ }^{1, *}$ C. Hidalgo,${ }^{1}$ T. Happel, ${ }^{2}$ and P. H. Diamond ${ }^{3}$ \\ ${ }^{1}$ Laboratorio Nacional de Fusión. As. Euratom-CIEMAT, 28040, Madrid, Spain \\ ${ }^{2}$ Max-Planck-Institut für Plasmaphysik, As. Euratom-IPP, 85748 Garching, Germany \\ ${ }^{3}$ University of California, San Diego, La Jolla, CA 92093-0424, U.S.A.
}

(Dated: November 1, 2011)

\begin{abstract}
The spatio-temporal behavior of the interaction between turbulence and flows has been studied close to the $\mathrm{L}-\mathrm{H}$ transition threshold conditions in the edge region $(\rho \geq 0.7)$ of TJ-II plasmas. The temporal dynamics of the interaction displays an oscillatory behaviour with a characteristic predator-prey relationship. The spatial evolution of this turbulence-flow oscillation-pattern has been measured, for the first time, showing both, radial outward and inward propagation velocities of the turbulence-flow front. The results indicate that the edge shear flow linked to the $\mathrm{L}-\mathrm{H}$ transition can behave either as a slowing-down, damping mechanism of outward propagating turbulent-flow oscillating structures, or as a source of inward propagating turbulence-flow events.
\end{abstract}

The High confinement mode $(\mathrm{H}$-mode) regime has been extensively studied since its discovery in the ASDEX tokamak [1]. Although significant progress has been made in describing the transition, the physical mechanism triggering the $\mathrm{H}$-mode has still not been clearly identified. Bifurcation theory models based on the coupling between turbulence and radially sheared $E \times B$ flows (sheared flows) describes the Low to High confinement mode transition ( $\mathrm{L}-\mathrm{H}$ transition) passing through an intermediate, oscillatory transient stage $[2,3]$. These models consist of coupled evolution equations for turbulence, sheared flow and pressure gradient. Using the input power as a control parameter for the pressure gradient, these dynamical systems evolve from $\mathrm{L}-$ to $\mathrm{H}-$ mode. By increasing the pressure gradient, the instability grows until it is damped by the self-generated sheared flows. The transition occurs when the turbulence driven sheared flow is high enough to overcome the flow damping. As it is discussed in Ref. [3], by including the evolution of zonal flows self-consistently, the critical input power for the transition is lowered. Further studies [4] show that zonal flows are a necessary step for the transition. Zonal flows trigger the transition by regulating the turbulence until the mean shear flow is high enough to suppress turbulence effectively, which in turn subsequently impedes the zonal flow generation. Due to the self-regulation between turbulence and flows, the transition is marked by an oscillatory behavior with a characteristic predator-prey relationship [5] between turbulence and zonal flows.

This intermediate oscillatory transient stage has been seen in the $\mathrm{L}-\mathrm{H}$ transition experiments in several devices. A dynamic behaviour of plasma parameters occurring near the $\mathrm{L}-\mathrm{H}$ transition threshold conditions was first observed in the $\mathrm{H}-1$ stellarator [6] and later in the DIII-D tokamak $[7,8]$ where experimental evidence was found supporting the predator-prey relationship between turbulence and flows as the basis for the $\mathrm{L}-\mathrm{H}$ transition. Recently, this intermediate stage has also been observed in other devices: NSTX [9], TJ-II [10], AUG [11] and EAST [12].

In these experiments, as in the predator-prey theory model [3], only the temporal dynamics of the turbulence-flow interaction is studied. Even though the spatial structure of the oscillating flow in the plasma edge region is shown in TJ-II [10] and AUG [11] and in a wider radial region in $\mathrm{H}-1[13,14]$, no information is given on its spatial evolution or spatial propagation. However, as it has been pointed out in Ref. [15], where the 0 -dimensional predator-prey theory model is upgraded toward a 1-dimensional one, the spatial evolution should also be taken into account as a necessary step to go towards the $\mathrm{L}-\mathrm{H}$ transition model.

The present work addresses for the first time this fundamental issue from the experimental point of view. To that end, dedicated experiments were carried out close to the $\mathrm{L}-\mathrm{H}$ transition threshold conditions to measure the spatial evolution of the oscillation-pattern of turbulence and flows. In this work, the radial propagation velocity of the turbulence-flow front measured during the oscillatory transient stage in the vicinity of the edge radial electric field shear location is described along with the radial distribution of the turbulence-flow oscillation-pattern. The results are discussed in terms of a mutual influence of the turbulence-flow oscillation-pattern and the edge shear-flow.

The experiments have been carried out in the TJ-II stellarator (magnetic field $B_{T}=0.95 \mathrm{~T}$, plasma minor and major radius $\langle a\rangle \leq 0.22 \mathrm{~m}$ and $R=1.5 \mathrm{~m}$, respectively), in pure NBI-heated plasmas (line-averaged plasma density $\left\langle n_{e}\right\rangle=2-4 \times 10^{19} \mathrm{~m}^{-3}$, central electron temperature $T_{e}=300-400 \mathrm{eV}$ ). The NBI input heating power is kept constant along the discharge at 500 $\mathrm{kW}$ and the fraction of NBI absorbed power - estimated using the FAFNER2 code that takes into account shine through, CX and ion losses- increases from 55 to $70 \%$ as the plasma density rises. TJ-II has a low, negative magnetic shear and the possibility to modify the rotational transform $\iota / 2 \pi$ within a rather broad range from 0.9 to 
2.2. In these experiments the magnetic configuration is kept constant, the rotational transform at the plasma edge being equal to 1.53. As expected, consequence of the high rotational transform, no GAM oscillations have been detected in TJ-II to date.

Due to the temporal and spatial scales involved in the $\mathrm{L}-\mathrm{H}$ transition physics, specific experimental techniques are required to experimentally investigate the turbulence and flows dynamics [16]. In TJ-II, a two-channel Doppler reflectometer is used that allows the measurement of the radial electric field, $E_{r}$ and density fluctuations at two radial positions simultaneously with good spatial and temporal resolution [17].

As reported in [10], close to the $\mathrm{L}-\mathrm{H}$ transition threshold conditions pronounced oscillations in both, $E_{r}$ and density fluctuation level are measured at the plasma edge, right inside the $E_{r}$-shear position but not outside. Depending on the NBI heating power and magnetic configuration, the oscillations can endure the whole NBI heating phase of the discharge. As an example, figure 1 shows the time evolution of line-averaged plasma density, the estimated NBI absorbed power, the plasma energy content and the $H_{\alpha}$ signal together with the spectrogram of the Doppler reflectometer signal measured at $\rho \approx 0.8$. At about $t \approx 170 \mathrm{~ms}$ the $H_{\alpha}$ drops, the plasma energy content and density increase and the spectrogram of the Doppler reflectometer signal shows an increase in its frequency and the onset of the oscillations. The oscillations appear as changes in the intensity and frequency of the Doppler reflectometry spectra, and show a predator-prey relationship between turbulence and flows, with the flow - the predator- following the turbulence - the prey- with a phase delay of $90^{\circ}$ in a limit-cycle way [10]. Contrary to DIII-D or AUG observations, in the TJ-II case no signatures of the oscillations appear in the $H_{\alpha}$ signals or in the evolution of plasma density and temperature, indicating a very small effect, if any, on particle transport. The oscillations are not detectable either in the Mirnov coils signals.

The repetition frequency of the oscillation-pattern changes along the plasma discharge as the line density increases. Besides, as the plasma density increases and the repetition frequency drops, the $E_{r}$ oscillation amplitude decreases while that of the density fluctuation level increases. These observations can be explained based on the collisional damping of flows which eventually sets the turbulence level [18].

In these experiments particular attention has been paid to the spatio-temporal evolution of plasma turbulence and flows in the vicinity of the $E_{r}$-shear position. The radial profile of $E_{r}$ changes from a rather flat profile in $\mathrm{L}$-mode in the radial range from $\rho=0.60$ to 0.85 to a sheared one during the oscillating phase with the $E_{r}$-shear located at $\rho \approx 0.82$. The $E_{r}$ radial profiles are shown in figure 2 . In addition to the $E_{r}$ profile measured in $\mathrm{L}$-mode, the extreme values of the $E_{r}$ oscillation mea-

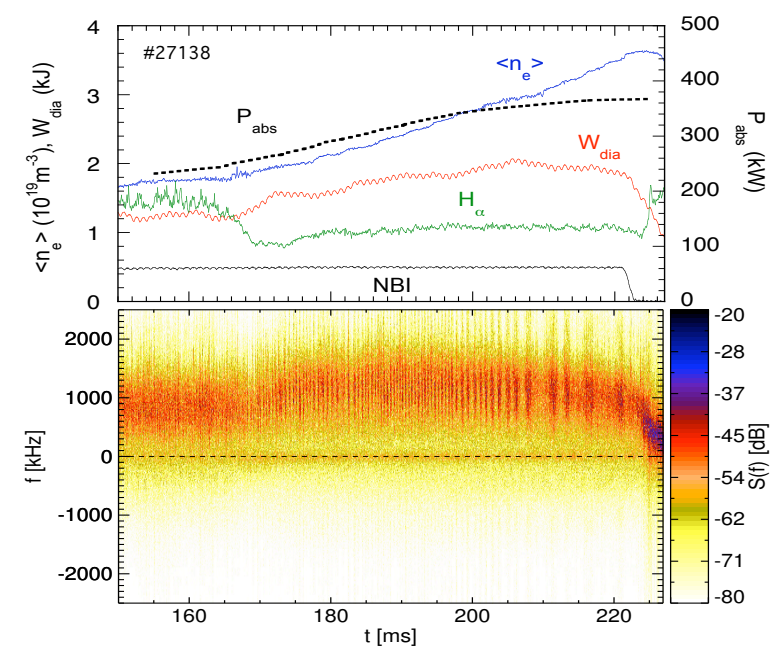

FIG. 1. The time evolution of line-averaged plasma density, estimated NBI absorbed power, plasma energy content and $H_{\alpha}$ signal (top) and spectrogram of the Doppler reflectometer signal measured at $\rho \approx 0.8$ (bottom). At $t \approx 170 \mathrm{~ms}$ the $H_{\alpha}$ drops, the frequency of the Doppler reflectometer signal increases and the oscillations start

sured at each radial position are also displayed. Large and small symbols are used to separate the oscillations measured at line-densities between $2-2.5 \times 10^{19} \mathrm{~m}^{-3}$ and close to $3 \times 10^{19} \mathrm{~m}^{-3}$. The $E_{r}$ oscillation amplitude is about $1 \mathrm{kV} / \mathrm{m}$ close to the $E_{r}$-shear and increases gradually as inner radial positions are probed. As a consequence, the $E_{r}$ well of about $10 \mathrm{kV} / \mathrm{m}$ measured at the maximum of the oscillations shrinks in each limit-cycle and an inner shear layer is measured at $\rho \approx 0.75$ (see blue profile in figure 2).

The amplitude of the $E_{r}$ oscillations is shown in figure 3 together with the amplitude of the oscillations in the density fluctuation level, i.e. $r m s\left(\tilde{n}_{e}\right)$. While the $E_{r}$ oscillation amplitude decreases towards the $E_{r}$-shear position $(\rho \approx 0.82)$, the $\operatorname{rms}\left(\tilde{n}_{e}\right)$ oscillation amplitude is maximum right inside the $E_{r}$-shear position and tends to decrease towards inner radial positions.

As already stated, the TJ-II Doppler reflectometer allows measuring simultaneously at two radial positions which can be independently selected [19]. Therefore, it is possible to obtain information on the radial propagation characteristics of the cyclic spatio-temporal pattern, extending previous temporal $(0-\mathrm{D})$ characterization to a spatio-temporal $(1-\mathrm{D})$ one. An example is shown in figure 4. It displays the time evolution of both, density fluctuation level (figure 4.a) and $E_{r}$ (figure 4.b) measured simultaneously at $\rho=0.8$ and $\rho=0.75$. In addition, the time evolution of $E_{r}$-shear calculated from the data shown in figure 4.b is shown in figure 4.c. The relation between $E_{r}$-shear and density fluctuation level showing a limit-cycle behaviour is shown in figure 4.d. Pronounced changes in $E_{r}$-shear appear linked to the os- 


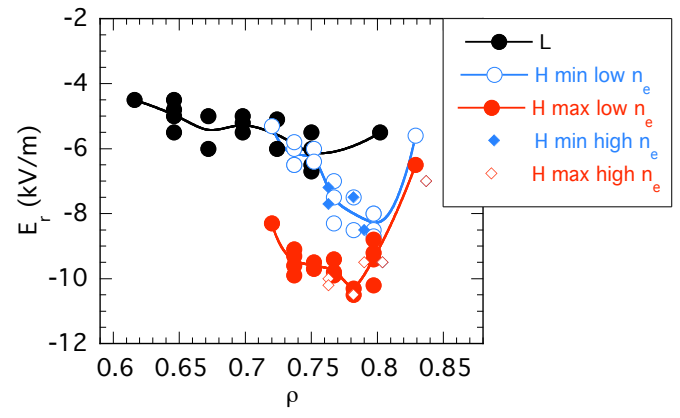

FIG. 2. $E_{r}$ profiles measured at the L-mode (black) and during the intermediate oscillatory phase; represented are the $E_{r}$ maxima (red) and minima (blue) measured during time intervals with line-densities between $2.0-2.5 \times 10^{19} \mathrm{~m}^{-3}$ (large symbols) and close to $3 \times 10^{19} \mathrm{~m}^{-3}$ (small symbols).

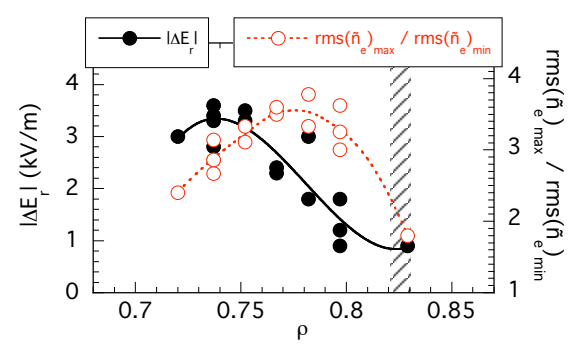

FIG. 3. Amplitude of the $E_{r}$ and $r m s\left(\tilde{n}_{e}\right)$ oscillations vs. plasma radius, measured during time intervals with line-densities between $2-2.5 \times 10^{19} \mathrm{~m}^{-3}$. The striped area indicates the radial location of the $E_{r}$-shear.

cillations in $\operatorname{rms}\left(\tilde{n}_{e}\right)$. Furthermore, a delay between the two channels can be seen indicating a radial propagation from the inner to the outer channel. This outward propagation is found in all the oscillation-patterns measured at line-densities between $2-2.5 \times 10^{19} \mathrm{~m}^{-3}$. At densities above $3 \times 10^{19} \mathrm{~m}^{-3}$, in some particular cases the propagation direction eventually reverses after a short time period without oscillations.

The analysis of the delays yields propagation velocities within the range $\approx 50-200 \mathrm{~m} / \mathrm{s}$ with a radial trend as shown in figure 5 . In this figure the vertical bars represent the error in the estimation of the propagation velocity and the horizontal ones represent the radial separation between the two channels in each discharge. The radial propagation velocity decreases as the oscillation-pattern approaches the $E_{r}$-shear position (at $\rho \approx 0.82$ ). The inward propagation velocities are also included in figure 5. Similar values are obtained although no clear radial dependence can be inferred. Similarly, as can be seen in figure 2 , the extreme values of the $E_{r}$ oscillations are comparable in both cases, for inward and outward propagating oscillation-patterns. However, the time evolution shows differences when comparing both cases. As the oscillation-pattern propagates outwards, the increase in the turbulence level produces an increase in the shearing

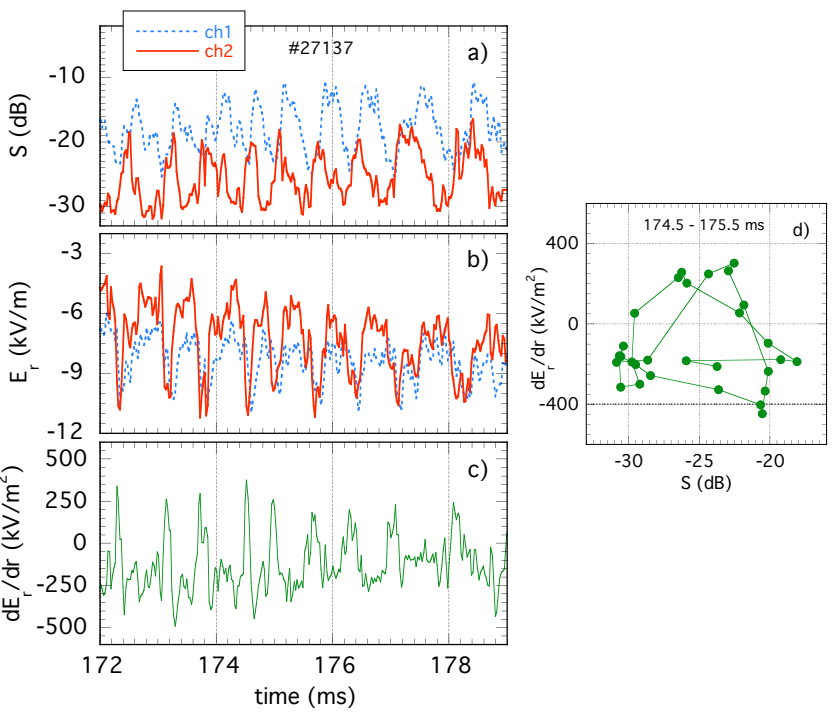

FIG. 4. The time evolution of (a) density fluctuation level and (b) $E_{r}$, measured simultaneously at $\rho=0.8$ (channel 1 in blue) and $\rho=0.75$ (channel 2 in red). (c) the time evolution of $E_{r}$-shear and (d) relation between $E_{r}$-shear and density fluctuation level (only two cycles are displayed).

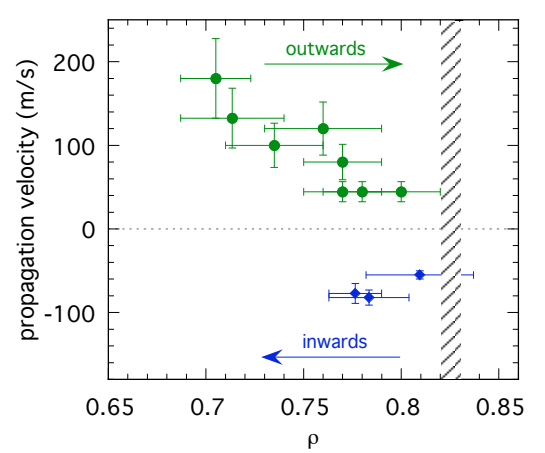

FIG. 5. Radial propagation velocity vs. plasma radius. The outward propagation velocity decreases as the $E_{r}$-shear location (at $\rho \approx 0.82$ ) is approached. Inward propagation velocities are represented using small symbols (in blue). The striped area indicates the radial location of the $E_{r}$-shear.

rate at the inner shear layer. This can be seen in figure 4: at each oscillation-cycle, the increase in the fluctuation level is follow by a decrease of $\left|E_{r}\right|$ what results in an increase in the negative $E_{r}$-shear. Oscillation-patterns propagating inwards show different dynamics. An example is shown in figure 6: the increase in the turbulence level is follow by an increase of $\left|E_{r}\right|$ what results in an increase in the outer positive $E_{r}$-shear.

In the reported TJ-II experiments, the spatio-temporal structure of the turbulence-flow interaction has been measured showing two distinct spatio-temporal patterns, namely outward and inward propagation.

The outward radial propagation velocity is close to $200 \mathrm{~m} / \mathrm{s}$ at the innermost radial positions $(\rho \approx 0.7)$ and gradually decreases to $50 \mathrm{~m} / \mathrm{s}$ as the oscillation-pattern 


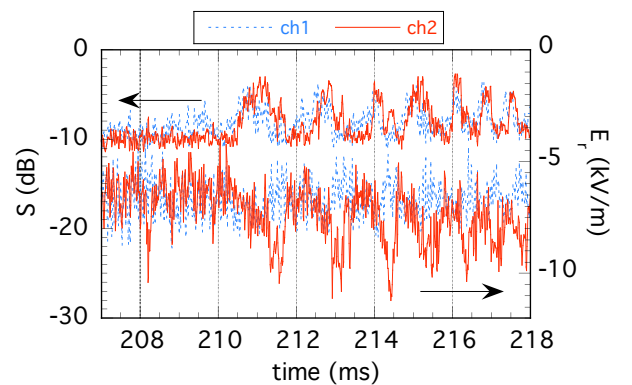

FIG. 6. The time evolution of density fluctuation level and $E_{r}$ measured simultaneously at $\rho=0.81$ (channel 1 in blue) and $\rho=0.77$ (channel 2 in red)

reaches the $E_{r}$-shear position. In each oscillation cycle the $E_{r}$-well shrinks and an inner shear layer develops at $\rho \approx 0.75$. A possible explanation for the spatiotemporal evolution of the oscillation-pattern could be linked to the radial spreading of the plasma turbulence from the plasma core to the edge barrier. Previous experiments performed in TJ-II have shown signatures of radial spreading of the turbulence as the plasma approaches the $\mathrm{H}-\mathrm{L}$ back transition conditions [20]. In those experiments the radial spreading of the turbulence produces a gradual retreat of the transport barrier and, as in the simulation results reported in [21], the key quantity to the control of turbulence spreading is the $E_{r}$-shearing rate. Similarly, in the present experiments, as the turbulence propagates towards the barrier, the associated turbulence driven flow generates the inner shear layer which in turn regulates the turbulence level. The present observation could be also figured out in terms of turbulent bursts propagating toward the plasma edge. These turbulent bursts could be generated in the plasma interior due to instabilities linked, for instance, to the magnetic topology. To explain the present experimental observations, each turbulent burst should be accompanied on its way to the plasma edge by a sheared-flow layer.

The inward propagation velocity of the oscillation-pattern is observed in some particular cases at the final stage of the discharge after a quiet period without oscillations. In those cases the oscillation-pattern arises at the outer shear layer position and propagates towards the plasma interior.

The present experimental results indicate that the edge shear flow linked to the $\mathrm{L}-\mathrm{H}$ transition can behave either as a slowing-down, damping mechanism of outward propagating turbulent-flow oscillating structures, or as a source of inward propagating turbulence-flow events. The first observation resembles simulations results reported in [22], where the influence of shear flows on radially propagating turbulent transport events is investigated showing the damping of the transport events at the center of the barrier. Some similarities also arise from the comparison of our results and those recently found in a linear plasma device and reported in [23]. Those ex- perimental results show how vortex-like structures propagating towards the plasma edge are slowed down and finally absorbed into the edge shear layer, transferring their momentum and energy to the shear flow which in turn is amplified. In our experiments, the deceleration in the oscillation-pattern propagation as it approaches the edge shear layer together with its absence at outer radial positions suggest an absorption process at the $E_{r}$-shear layer. In this process, the turbulence-flow events generate a dual shear layer, and thus enhance the formation of the $E_{r}$-well. It is interesting also to note that the buildup of the shear layer could be relevant as a source mechanism for intrinsic toroidal rotation [24].

The inward propagation of turbulence driven flows has also been observed in simulation studies [25]. In those studies, turbulence driven toroidal flows propagate both, radially inwards and outwards, whereas in our case, the turbulence-flow events generated at the inner side of the $E_{r}$-shear position propagate only inwards. Outside the $E_{r}$-shear position these events are not detected indicating the blocking effect of the barrier on the turbulent-flow events propagation.

In conclusion, the spatio-temporal evolution of the turbulence-flow interaction has been measured during the $\mathrm{L}-\mathrm{H}$ transition in a toroidal plasma. The turbulence-flow front propagation and its interaction with the edge shearflow have been described. The reported results show the need of approaching L-H transition studies within a 1dimensional spatio-temporal framework.

The authors acknowledge the entire TJ-II team for their support during the experiments. This work has been partially funded by the Spanish Ministry of Science and Innovation under contract number ENE2010-18409.

* e-mail address: teresa.estrada@ciemat.es

[1] F. Wagner et al., Phys. Rev. Lett. 49, 1408 (1982).

[2] P. H. Diamond, Y.-H. Liang, B. A. Carreras, and P. W. Terry, Phys. Rev. Lett. 72, 2565 (1994).

[3] E.-J. Kim and P. H. Diamond, Phys. Plasmas 10, 1698 (2003).

[4] M. A. Malkov and P. H. Diamond, Phys. Plasmas 16, 012504 (2009).

[5] V. Volterra, Nature 2972, 558 (1926).

[6] D. L. Rudakov, M. G. Shats, J. H. Harris, and B. D. Blackwell, Plasma Physics and Controlled Fusion 43, 559 (2001).

[7] R. J. Colchin et al., Phys. Rev. Lett. 88, 255002 (2002).

[8] R. J. Colchin et al., Nuclear Fusion 42, 1134 (2002).

[9] S. J. Zweben et al., Phys. Plasmas 17, 102502 (2010).

[10] T. Estrada et al., EPL (Europhysics Letters) 92, 35001 (2010).

[11] G. D. Conway et al., Phys. Rev. Lett. 106, 065001 (2011).

[12] G. S. Xu et al., Phys. Rev. Lett. 107, 125001 (2011).

[13] H. Punzmann and M. G. Shats, Phys. Rev. Lett. 93, 125003 (2004).

[14] H. Xia, M. G. Shats, and H. Punzmann, Phys. Rev. Lett. 
97, 255003 (2006).

[15] K. Miki and P. H. Diamond, Towards a $1 D$ model of $L$ I-H evolution dynamics (Asia Pacific Transport Working Group, Japan, 2011).

[16] T. Estrada et al., Plasma Phys. Control. Fusion 51, 124015 (2009).

[17] T. Happel et al., Rev. Sci. Instrum. 80, 073502 (2009).

[18] Z. Lin et al., Phys. Rev. Lett. 83, 3645 (1999).

[19] L. Cupido, J. Sánchez, and T. Estrada, Rev. Sci. Instrum. 75, 3865 (2004)
[20] T. Estrada, C. Hidalgo, and T. Happel, Nuclear Fusion 51, 032001 (2011).

[21] W. X. Wang et al., Phys. Plasmas 14, 072306 (2007).

[22] P. Beyer, S. Benkadda, X. Garbet, and P. H. Diamond, Phys. Rev. Lett. 85, 4892 (2000).

[23] M. Xu et al., Phys. Rev. Lett. 107, 055003 (2011).

[24] Ö. D. Gürcan, P. H. Diamond, T. S. Hahm, and R. Singh, Phys. Plasmas 14, 042306 (2007).

[25] W. Wang et al., Phys. Plasmas 17, 072511 (2010). 